\title{
Phase-difference operator
}

\author{
A. Luis and L.L. Sánchez-Soto \\ Departamento de Optica, Facultad de Ciencias Físicas, Universidad Complutense, 28040 Madrid, Spain
}

(Received 15 March 1993)

\begin{abstract}
We introduce a unitary operator representing the exponential of the phase difference between two modes of the electromagnetic field. The eigenvalue spectrum has a discrete character that is fully analyzed. We relate this operator with a suitable polar decomposition of the Stokes parameters of the field, obtaining a natural classical limit. The cases of weakly and highly excited states are considered, discussing to what extent it is possible to talk about the phase for a single-mode field. This operator is applied to some interesting two-mode fields.
\end{abstract}

PACS number(s): 42.50.Dv, 03.65.- $\mathrm{w}$

\section{INTRODUCTION}

The problem of a correct definition in quantum mechanics of the phase variable has a long history and has provoked many discussions [1]. Given the relevance of such a variable, there have been numerous attempts to solve this problem and certainly very interesting progress has been done in the last years [2-4].

A few experiments [5-7] have been also reported in which phase fluctuations for a monomode laser were measured, and attempts have been made to test some of the definitions [8-10], although unfortunately no clear conclusion emerged [11].

However, most of this work has been devoted to the properties of the phase operator for a one-mode quantum field or, equivalently, for a single harmonic oscillator. In this case, the absence of a proper phase operator is usually ascribed to the semiboundedness of the eigenvalue spectrum of the number operator.

On the other hand, from a practical point of view an absolute phase has no meaning and all measurements must be made relative to the phase of a reference system. Therefore, it seems that the most proper way to deal with the phase should be as a phase difference between the state considered and some reference phase state. One could think that this phase-difference operator should be just the difference of phase operators for each field as it were a position or momentum difference. But, given the periodic character of this variable, this statement must be taken with care.

It is worth mentioning that the variable canonically conjugated to the phase-difference operator is the number difference, that is not bounded from below. So, it is reasonable to expect the existence of a phase-difference operator free from the problems arising in the one-mode case. This is to say, it is possible to have a definite phase difference between two independent oscillators for a fixed number of quanta in the two systems.

Moreover, if a phase measurement must be thought of as a measurement of the phase difference, this phasedifference operator could give to what extent and in which terms we can talk about the phase of a onedimensional system.
The aim of the present paper is just to show how to introduce a well-behaved phase-difference operator and to study its main properties.

\section{PHASE-DIFFERENCE OPERATOR}

\section{A. Polar decomposition of the amplitudes of two oscillators}

Our purpose here is to find an operator $E_{12}$ exponential of the phase difference between two independent oscillators like two modes of the electromagnetic field.

Let $E_{1}$ and $E_{2}$ be the Susskind-Glogower phase operators [12] for the two oscillators. Given the classical expression $\exp i\left(\phi_{1}-\phi_{2}\right)=\exp \left(i \phi_{1}\right) \exp \left(-i \phi_{2}\right)$, we may be tempted to introduce a relative exponential phase operator as $E_{1} E_{2}^{\dagger}$, that commutes with the total-number operator $N=N_{1}+N_{2} ; N_{1}$ and $N_{2}$ being the number operators for each mode. Unfortunately, $E_{1} E_{2}^{\dagger}$ annihilates the state $|0, n\rangle \equiv|0\rangle \otimes|n\rangle$ in the subspace with fixed total number of quanta $n$, and this precludes the existence of eigenstates for this operator. This is an obvious consequence of the lack of unitarity of $E_{1}$ and $E_{2}$.

In order to avoid this problem, let us try a polar decomposition of the complex amplitudes of the two modes as

$$
a_{1} a_{2}^{\dagger}=E_{12} \sqrt{N_{1}\left(N_{2}+1\right)},
$$

where $a_{1}$ and $a_{2}$ are the annihilation operators for both modes. We shall see that Eq. (2.1) has unitary solutions for $E_{12}$; that is, $E_{12}=\exp \left(i \Phi_{12}\right), \Phi_{12}$ being the Hermitian relative-phase operator.

As in the one-dimensional case, the polar decomposition does not completely define the exponential of phase. In our case the matrix elements $\left\langle n_{1}, 0\left|E_{12}\right| 0, n_{2}\right\rangle$ are undefined and thus $E_{12}$ cannot be uniquely determined by the unitarity requirement. We must impose then further conditions, the most adequate being the commutation relations.

For a classical harmonic oscillator the action $(j)$ and phase $(\phi)$ variables verify the fundamental Poisson 
bracket

$$
\{j, \phi\}=1,
$$

so, for two independent oscillators we have

$$
\begin{aligned}
\left\{j_{1}+j_{2}, \phi_{12}\right\} & =0, \\
\left\{\left(j_{1}-j_{2}\right) / 2, \phi_{12}\right\} & =1,
\end{aligned}
$$

where $\phi_{12}$ is the classical phase difference. With the standard prescription that operator commutators are related to classical Poisson brackets via

$$
[u, v] \leftrightarrow i \hbar\{u, v\}
$$

we have that the quantum counterpart of $\phi_{12}$ commutes with the total number operator. Thus the commutator associated with the second Poisson bracket should be verified on the finite-dimensional spaces with fixed total number $n$, but this is not possible.

The quantum translation of (2.3) in terms of the exponential of phase difference is

$$
\begin{gathered}
{\left[E_{12}, N_{1}+N_{2}\right]=0,} \\
{\left[E_{12},\left(N_{1}-N_{2}\right) / 2\right]=E_{12},}
\end{gathered}
$$

the second one can be recognized as the analogous of the well-known Lerner criterion [13].

It should be noted that there is a nonunitary solution for $E_{12}$ verifying simultaneously these two commutation relations, namely the Susskind-Glogower phase-difference operator $E_{1} E_{2}^{\dagger}$. The lack of unitarity of this solution is a reminiscence of the incompatibility in the quantum translation of (2.3). Recently Ban [14] and Hradil [15] have introduced a unitary exponential of phase operator verifying $(2.5 \mathrm{~b})$ but neither (2.5a) nor a polar decomposition. Therefore, we shall consider the polar decomposition (2.1) together with the condition (2.5a), which leads to a unique (up to an arbitrary global phase) unitary solution for (2.1).

Instead of solving Eq. (2.1) directly, we can take advantage of the fact that the operators [16]

$$
\begin{aligned}
& J_{x}=\frac{1}{2}\left(a_{1}^{\dagger} a_{2}+a_{2}^{\dagger} a_{1}\right), \\
& J_{y}=\frac{i}{2}\left(a_{2}^{\dagger} a_{1}-a_{1}^{\dagger} a_{2}\right), \\
& J_{z}=\frac{1}{2}\left(a_{1}^{\dagger} a_{1}-a_{2}^{\dagger} a_{2}\right),
\end{aligned}
$$

satisfy the commutation relations for the Lie algebra of the three-dimensional rotation group $\mathrm{SU}(2)$ :

$$
\left[J_{k}, J_{l}\right]=i \epsilon_{k l m} J_{m}
$$

The Casimir invariant for this group can be put into the form

$$
\mathbf{J}^{2}=\frac{N}{2}\left(\frac{N}{2}+1\right)
$$

in fact $N$ commutes with all the operators in (2.6).

The total Hilbert space of the problem $\mathcal{H}_{1} \otimes \mathcal{H}_{2}$ can be expressed then as direct sum of the subspaces invariant under these angular momentum operators,

$$
\mathcal{H}_{1} \otimes \mathcal{H}_{2}=\bigoplus_{n=0}^{\infty} \mathcal{H}_{n}
$$

Each $\mathcal{H}_{n}$ having fixed total number $n$ is spanned by the $2 j+1=n+1$ vectors $|j, m\rangle$, simultaneous eigenstates of $\mathbf{J}^{2}$ and $J_{z}$. The number eigenstates $\left|n_{1}, n_{2}\right\rangle$ in $\mathcal{H}_{n}$ correspond to the $|j, m\rangle$ basis as follows:

$$
\left|n_{1}, n_{2}\right\rangle=\left|j=\left(n_{1}+n_{2}\right) / 2, m=\left(n_{1}-n_{2}\right) / 2\right\rangle .
$$

From Eq. (2.6) one gets immediately that

$$
\begin{aligned}
& J_{+}=J_{x}+i J_{y}=a_{1}^{\dagger} a_{2}, \\
& J_{-}=J_{x}-i J_{y}=a_{1} a_{2}^{\dagger},
\end{aligned}
$$

and then Eq. (2.1) can be recast as

$$
J_{-}=E_{12} \sqrt{J_{+} J_{-}} .
$$

Since the operator $E_{12}$ commutes with the total number $N$, we may rather study its restriction to each subspace $\mathcal{H}_{n}$. Calling $E_{12}^{(n)}$ this restriction, Eq. (2.12) can be easily solved obtaining the unitary $\mathrm{SU}(2)$ exponential of phase operator [17-19]

$$
E_{12}^{(n)}=\sum_{m=-j+1}^{j}|j, m-1\rangle\left\langle j, m\left|+e^{i(n+1) \phi^{(n)}}\right| j, j\right\rangle\langle j,-j|,
$$

$\phi^{(n)}$ being an arbitrary phase. Note that the crucial extra term in Eq. (2.13), which establishes the unitarity of $E_{12}^{(n)}$, is precisely based on the finite number of states. For the harmonic-oscillator operators such decomposition is unattainable because the number states are extended to infinity and no extra term projecting the upper state to ground state exists (unless one truncates, as in the PeggBarnett approach, the infinite ladder of the oscillator and creates an upper state). Therefore, in each subspace $\mathcal{H}_{n}$ there are $n+1$ orthonormal states verifying that

$$
E_{12}^{(n)}\left|\phi_{r}^{(n)}\right\rangle=e^{i \phi_{r}^{(n)}}\left|\phi_{r}^{(n)}\right\rangle
$$

with $r=0, \ldots, n$. These states can be expressed in the number basis as

$$
\left|\phi_{r}^{(n)}\right\rangle=\frac{1}{\sqrt{n+1}} \sum_{n_{1}=0}^{n} e^{i n_{1} \phi_{r}^{(n)}}\left|n_{1}, n-n_{1}\right\rangle
$$

where, by taking the same $2 \pi$ window in each subspace, we have

$$
\phi_{r}^{(n)}=\phi_{0}+\frac{2 \pi r}{n+1}
$$


The expression for $E_{12}$ on the whole space is just made of infinity many copies of the SU(2) exponential of phase operator

$$
\begin{aligned}
E_{12}= & \sum_{n=0}^{\infty} E_{12}^{(n)}=\sum_{n=0}^{\infty} \sum_{r=0}^{n}\left|\phi_{r}^{(n)}\right\rangle e^{i \phi_{r}^{(n)}}\left\langle\phi_{r}^{(n)}\right| \\
= & \sum_{n=0}^{\infty} \frac{1}{n+1} \sum_{r=0}^{n} \sum_{k, k^{\prime}=0}^{n}|k, n-k\rangle e^{i\left(k-k^{\prime}+1\right) \phi_{r}^{(n)}} \\
& \times\left\langle k^{\prime}, n-k^{\prime}\right|
\end{aligned}
$$

which is very reminiscent of the operator introduced by Lévy-Leblond [20] in a different way.

Since $E_{12}$ is unitary, it defines a Hermitian phasedifference operator

$$
\Phi_{12}=\sum_{n=0}^{\infty} \sum_{r=0}^{n}\left|\phi_{r}^{(n)}\right\rangle \phi_{r}^{(n)}\left\langle\phi_{r}^{(n)}\right|
$$

and we have $E_{12}=\exp \left(i \Phi_{12}\right)$.

This operator differs from other approaches, mainly because it cannot be obtained from a previous construction of phase operators for the individual oscillators involved. Unlike in the Susskind-Glogower approach, $E_{12}$ is unitary.

On the other hand $\Phi_{12}$ has discrete eigenvalues (for each subspace $\mathcal{H}_{n}$ there are $n+1$ uniformly distributed in the interval $[0,2 \pi])$, in comparison with other formalisms $[21,22]$ that give continuous spectrum in the interval $[0,4 \pi]$.

The situation presents the same qualitative features as in the Carruthers and Nieto definition of phase difference by means of relative sine and cosine operators [1]. However, in this approach of Carruthers and Nieto, the eigenvalues of the phase difference are in the upper half circle for the cosine case, and on the right one in the sine case.

In the limit of high $n$ this spectrum becomes dense, as might be expected. But, the case with $n=0$ is especially relevant, since the state $|0,0\rangle$ is an eigenstate of $E_{12}$. The unitarity requirement makes the corresponding eigenvalue to be an arbitrary phase.

In the next section we shall try to understand this behavior (in both limits of small and high $n$ ) by means of a simple relative phase-sensitive arrangement for the electromagnetic field.

\section{B. Stokes parameters}

In order to gain physical insight into the previous polar decomposition, we shall relate it to the Stokes parameters. It is well known that in classical optics to characterize the polarization ellipse of two orthogonal oscillations of the same frequency, three independent quantities are necessary: the two amplitudes and the phase difference. For practical purposes, it is customary to characterize the resultant oscillation by the Stokes parameters, which are directly measurable quantities [23].

In the quantum treatment of the two-mode field considered here, the following Hermitian Stokes operators can be defined [24]:

$$
\begin{aligned}
& S_{0}=\left(a_{1}^{\dagger} a_{1}+a_{2}^{\dagger} a_{2}\right), \\
& S_{1}=\left(a_{1}^{\dagger} a_{2}+a_{2}^{\dagger} a_{1}\right), \\
& S_{2}=i\left(a_{2}^{\dagger} a_{1}-a_{1}^{\dagger} a_{2}\right), \\
& S_{3}=\left(a_{1}^{\dagger} a_{1}-a_{2}^{\dagger} a_{2}\right) .
\end{aligned}
$$

Note that, apart from a factor of 2 , the operators $S_{i}$ $(i=1,2,3)$ coincide with the operators (2.6) of an angular momentum, while $S_{0}$ represents the total number $N$. The noncommutability of the Stokes operators precludes the simultaneous measurement of the physical quantities represented by these operators. The $S_{i}$ may be viewed as the generators of a group of transformations locally isomorphic to the three-dimensional rotation group and which leave the operator $S_{0}$ invariant. This may be considered as the sound basis for the close relation between Stokes parameters and the Poincaré sphere introduced in the classical description of light. Note that the eigenstates of $S_{0}$ belong to spaces which under the action of rotations transform according to some irreducible representation of this group.

In order to show that the operators $(2.19)$ are the ana$\log$ of the classical Stokes parameters, let us compute their mean values for a two-mode coherent state

$$
\left|\alpha_{1}, \alpha_{2}\right\rangle=e^{-\left(\left|\alpha_{1}\right|^{2}+\left|\alpha_{2}\right|^{2}\right) / 2} \sum_{n_{1}, n_{2}=0}^{\infty} \frac{\alpha_{1}^{n_{1}} \alpha_{2}^{n_{2}}}{\sqrt{n_{1} ! n_{2} !}}\left|n_{1}, n_{2}\right\rangle
$$

that has special significance in describing the classical limit of the system. It is easy to get [25]

$$
\begin{aligned}
& s_{0}=\left\langle\alpha_{1}, \alpha_{2}\left|S_{0}\right| \alpha_{1}, \alpha_{2}\right\rangle=\left|\alpha_{1}\right|^{2}+\left|\alpha_{2}\right|^{2}, \\
& s_{1}=\left\langle\alpha_{1}, \alpha_{2}\left|S_{1}\right| \alpha_{1}, \alpha_{2}\right\rangle=2\left|\alpha_{1}\right|\left|\alpha_{2}\right| \cos \left(\phi_{1}-\phi_{2}\right), \\
& s_{2}=\left\langle\alpha_{1}, \alpha_{2}\left|S_{2}\right| \alpha_{1}, \alpha_{2}\right\rangle=-2\left|\alpha_{1}\right|\left|\alpha_{2}\right| \sin \left(\phi_{1}-\phi_{2}\right), \\
& s_{3}=\left\langle\alpha_{1}, \alpha_{2}\left|S_{3}\right| \alpha_{1}, \alpha_{2}\right\rangle=\left|\alpha_{1}\right|^{2}-\left|\alpha_{2}\right|^{2},
\end{aligned}
$$

where $\alpha_{i}=\left|\alpha_{i}\right| \exp \left(i \phi_{i}\right), \phi_{i}$ being the classical phase of the state. It is evident that (2.21) are exactly the Stokes parameters for two classical oscillations of amplitudes $\left|\alpha_{i}\right|$ and phases $\phi_{i}$.

As discussed in Ref. [26], for a given state of the twomode field, the classical polarization properties can be described by the coherency matrix that, in terms of the Stokes parameters, can be written as

$$
\begin{aligned}
J & =\left(\begin{array}{cc}
\left|\alpha_{1}\right|^{2} & \left|\alpha_{1}\right|\left|\alpha_{2}\right| e^{-i\left(\phi_{1}-\phi_{2}\right)} \\
\left|\alpha_{1}\right|\left|\alpha_{2}\right| e^{i\left(\phi_{1}-\phi_{2}\right)} & \left|\alpha_{2}\right|^{2}
\end{array}\right) \\
& =\frac{1}{2}\left(\begin{array}{cc}
s_{0}+s_{3} & s_{1}+i s_{2} \\
s_{1}-i s_{2} & s_{0}-s_{3}
\end{array}\right) .
\end{aligned}
$$


If we denote $s_{ \pm}=\left(s_{1} \pm i s_{2}\right) / 2$ then the classical phase difference between the two modes is unambiguously obtained as

$$
s_{+}=e^{-i\left(\phi_{1}-\phi_{2}\right)}\left|\alpha_{1}\right|\left|\alpha_{2}\right|=e^{-i\left(\phi_{1}-\phi_{2}\right)} \sqrt{s_{-} s_{+}} .
$$

The quantum analog of the separation of the complex amplitude into a real part and a phase factor is just (2.12), which seems to be a natural way to characterize the phase difference with a clear counterpart in the classical limit.

\section{LIMIT OF WEAKLY EXCITED STATES}

We shall try, in first place, to justify the discrete character of the phase difference in the quantum case, whose effects will be more evident in the limit of small number of photons.

Perhaps, the simplest arrangement sensitive to the relative phase is a homodyne detection $[27,28]$ schematized in Fig. 1. The beam splitter couples the input modes 1 and 2 transforming them into the output modes $\overline{1}, \overline{2}$ whose photon numbers $\bar{N}_{1}, \bar{N}_{2}$ are measured. Fluctuations in the modes therefore get coupled, which causes the appearance of intriguing behaviors.

For a linear, lossless, and passive beam splitter the number operators at the output can be expressed in terms of the operators $\mathbf{J}^{2}$ and $\mathbf{J}$, and so the action of the beam splitter can be visualized as the process of measuring the rotations of $\mathbf{J}[29]$. The parameters of such an action depends on the particular choice for the transmission and reflection coefficients of the beam splitter.

The lossless beam splitter conserves the total energy in the pair of modes and therefore we have

$$
\bar{N}_{1}+\bar{N}_{2}=N_{1}+N_{2} \text {. }
$$

Since $\bar{N}_{1}$ and $\bar{N}_{2}$ are linear combinations of $\mathbf{J}$ and $\mathbf{J}^{2}$, the fluctuations in the output number of photons are due to fluctuations in the amplitudes and in the phase difference of the incoming states, but not in their phase sum.

To focus on the behavior of the phase difference, we shall consider incident fields with nonfluctuating amplitudes. So, for definiteness, we consider the initial photon

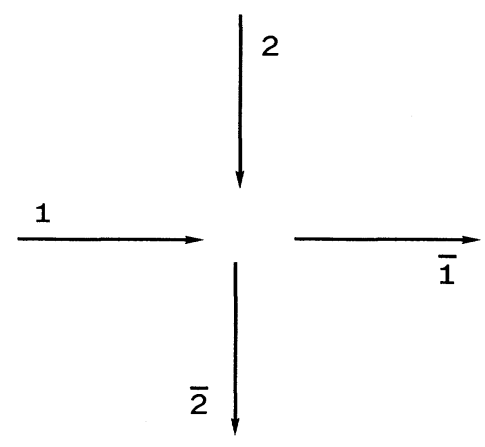

FIG. 1. Outline of the beam splitter geometry used in a phase-sensitive measurement of the electromagnetic field. states to be a product of number states $\left|n_{1}, n_{2}\right\rangle$ with $n=n_{1}+n_{2}$. Due to (3.1) the output state will be an eigenstate of $\bar{N}$. As it is well known, the photon numbers at the output are no longer sharply determined. The beam splitter coupling has brought about noise in the photon number of each mode, although the total photon number in both modes is invariant and free noise [30].

The state $\left|n_{1}, n_{2}\right\rangle$ transforms into a highly correlated superposition of states with $n$ total number of photons in the two modes, namely the states $|n, 0\rangle,|n-1,1\rangle, \mid n-$ $2,2\rangle, \ldots,|0, n\rangle$, and then the number of possible outcomes in the measurement of $\bar{N}_{1}$ and $\bar{N}_{2}$ are just $n+1$. Since we have considered the incident states with welldefined amplitudes, the number of outcomes can only depend on the number of possible values for the phase difference, so we can observe just $n+1$ values which is precisely the same number predicted by the phase-difference operator for a state in a $\mathcal{H}_{n}$ subspace, as it is the case.

Although there are reasons for requiring the phase difference between number-state fields to be completely random [21], in our approach only in the limit of $n_{1}$ or $n_{2}$ high enough is it to be expected that the measured phase difference is uniformly distributed over the interval $[0,2 \pi]$, and this is in agreement with the theoretical and experimental results of Noh, Fougères, and Mandel [11].

Moreover, when the incident state is $|0,0\rangle$, we have clearly just only one outcome. This is consistent with taking it as a phase-difference eigenstate. This strange situation could be understood considering its field fluctuations due only to the ones of the phase sum. This is equivalent to ascribe the field fluctuations of the vacuum in the one-mode case to the phase and not to the amplitude.

\section{LIMIT OF HIGHLY EXCITED STATES: PHASE FOR A ONE-DIMENSIONAL SYSTEM}

Actually, the more extended use of the arrangement discussed in the preceding section makes use of a very intense state of well-defined phase (for example a coherent state of high mean number of photons) in one of the incident modes, say 2. This scheme can be used to measure the properties of the field in mode 1 , and in our case, its phase properties.

Our aim is to study the behavior of the phase-difference operator by means of a suitable approximation for a high number of photons. Since we expect a continuous character, in this limit we can approximate the $r$ sum in expressions like (2.17) and (2.18) by an integral. For definiteness, we shall consider the operator $E_{12}^{\ell}$, and we have

$$
\frac{e^{i\left(\ell+k-k^{\prime}\right) \phi_{0}}}{n+1} \sum_{r=0}^{n} e^{i\left(\ell+k-k^{\prime}\right) \frac{2 \pi r}{n+1}}
$$

that will be replaced by

$$
\frac{1}{2 \pi} \int_{\phi_{0}}^{\phi_{0}+2 \pi} d \phi e^{i\left(\ell+k-k^{\prime}\right) \phi} .
$$

While the integral gives $\delta_{\ell+k-k^{\prime}, 0}$, the sum gives zero 
unless $\frac{\ell+k-k^{\prime}}{n+1}$ is an integer $m$, taking in this case the value $e^{i m \phi_{0}}$.

The approximation in replacing (4.1) by (4.2) is tantamount to considering that the contribution when $m \neq 0$ is negligible. Looking at (2.17) this will be the case when the spread of the photon number distribution is small compared with the mean value of the total photon number $n$, assumed high enough. This can be verified, for example, when just only one mode is in a coherent state of high mean photon number and the other one involves a small number of photons in comparison with the coherent one, but otherwise arbitrary. This condition is verified as well when the two modes are intense coherent states.

With all this in mind, this replacement gives an approximate expression for $E_{12}^{\ell}$ in the form

$$
E_{12}^{\ell} \simeq \int_{\theta_{0}}^{\theta_{0}+2 \pi} d \theta \int_{\phi_{0}}^{\phi_{0}+2 \pi} d \phi|\theta+\phi, \theta\rangle e^{i \ell \phi}\langle\theta+\phi, \theta|
$$

where $|\theta+\phi, \theta\rangle$ denotes a two-mode Susskind-Glogower phase state

$$
|\theta+\phi, \theta\rangle=\frac{1}{2 \pi} \sum_{n_{1}, n_{2}=0}^{\infty} e^{i n_{1}(\theta+\phi)} e^{i n_{2} \theta}\left|n_{1}, n_{2}\right\rangle .
$$

Therefore, in this limit we recover the equivalent version of the Susskind-Glogower phase-difference operator:

$$
E_{12}^{\ell} \simeq\left(E_{1} E_{2}^{\dagger}\right)^{\ell}
$$

and we lost the unitarity of the operator. Incidentally, we note that, for an arbitrary function $f$, we have

$$
f\left(E_{12}\right) \neq f\left(E_{1} E_{2}^{\dagger}\right) .
$$

As we can see, in this limit we get expressions for the phase difference resembling the ones from other approaches starting from a one-mode analysis. To discuss this resemblance we use (4.3) to obtain the mean value of a periodic function of phase difference $f\left(\Phi_{12}\right)$ on a state $|\psi\rangle=\left|\psi_{1}, \psi_{2}\right\rangle$,

$$
\begin{aligned}
\left\langle\psi\left|f\left(\Phi_{12}\right)\right| \psi\right\rangle \simeq \int_{\theta_{0}}^{\theta_{0}+2 \pi} d \theta \int_{\phi_{0}}^{\phi_{0}+2 \pi} & d \phi P_{1}(\phi+\theta) \\
& \times P_{2}(\theta) f(\phi),
\end{aligned}
$$

where $P(\theta)=|\langle\theta \mid \psi\rangle|^{2}$ is the phase probability distribution function of the Pegg-Barnett formalism for the one-mode case.

To some extent, expression (4.7) is an expected result, since it is valid for high photon numbers, and all phase approaches coincide in this limit. However, in the twomode case, this limit can be reached when only one of the modes is highly excited, while the other one can be one of few photons.

If we consider in mode 2 a state whose phase distribution $P_{2}(\theta)$ is narrow enough, we are in the proper conditions for the observation of the phase properties of mode
1 (for example, by means of the arrangement discussed in Sec. III), and the results obtained from $\Phi_{12}$ can be interpreted as information about the phase in mode 1 . Taking $P_{2}(\theta)$ to be an arbitrarily narrow function of $\theta$, centered for simplicity on the value $\phi_{0}$, we have from (4.7)

$$
\left\langle f\left(\Phi_{12}\right)\right\rangle=\int_{0}^{2 \pi} d \phi P_{1}(\phi) f\left(\phi-\phi_{0}\right) .
$$

What we get is nothing but the Pegg-Barnett phase approach for the one-mode case, which is often considered as giving the expected results, even for the vacuum. Ellinas [18] also derived similar results in an elegant way performing a polar decomposition of the $\mathrm{SU}(2)$ algebra and taking the group contraction in the limit $j \rightarrow \infty$.

Finally, we must stress (according with the comments made in the Introduction) that this procedure cannot be understood as giving a phase operator for the onemode problem. In fact, the Pegg-Barnett formalism, reproduced here in the limit of a practical observation of phase, does not give such operator in the infinite Hilbert space.

\section{PHASE DIFFERENCE PROPERTIES OF SOME TWO-MODE FIELD STATES}

\section{A. Two-mode coherent states}

Among other basic properties, coherent states have a special significance describing the classical limit of a system and they are the prototype for the radiation emitted by a classical current source [31].

Here we are going to study their phase-difference properties in the limit of high excitation, so we are in the conditions of application of (4.7) to the two-mode coherent state (2.20).

In the limit of large coherent amplitudes the $P_{i}(\theta)$ functions can be approximated by the Gaussian distributions $[3]$

$$
P_{i}(\theta)=\sqrt{\frac{2}{\pi}}\left|\alpha_{i}\right| \exp -\left(\frac{\left|\alpha_{i}\right|^{2}}{2}\left(\theta-\phi_{i}\right)^{2}\right),
$$

where $\alpha_{i}=\left|\alpha_{i}\right| e^{i \phi_{i}}$, and we can extend to $\pm \infty$ the limits of integration in (4.7) without significant error.

Then, we have

$$
\left\langle f\left(\Phi_{12}\right)\right\rangle=\int d \phi f(\phi) P(\phi),
$$

where $P(\phi)$ is the Gaussian distribution

$$
\begin{aligned}
P(\phi)= & {\left[\frac{2}{\pi\left(\frac{1}{\left|\alpha_{1}\right|^{2}}+\frac{1}{\left|\alpha_{2}\right|^{2}}\right)}\right]^{\frac{1}{2}} } \\
& \times \exp \left\{-\frac{2\left[\phi-\left(\phi_{1}-\phi_{2}\right)\right]^{2}}{\left(\frac{1}{\left|\alpha_{1}\right|^{2}}+\frac{1}{\left|\alpha_{2}\right|^{2}}\right)}\right\} .
\end{aligned}
$$

We finally get 


$$
\begin{aligned}
\left\langle\Phi_{12}\right\rangle & =\phi_{1}-\phi_{2}, \\
\left\langle\left(\Delta \Phi_{12}\right)^{2}\right\rangle & =\frac{1}{4\left|\alpha_{1}\right|^{2}}+\frac{1}{4\left|\alpha_{2}\right|^{2}}
\end{aligned}
$$

that is an expected result that could be obtained from a semiclassical analysis of fluctuations.

It can be recognized in $\left(\Delta \Phi_{12}\right)^{2}$ the sum of the uncertainties of the individual phases. This could be obtained in many ways, since all phase formalisms coincide in this limit, including the one described here simply by taking $\left|\alpha_{1}\right| \rightarrow \infty$ and $\left|\alpha_{2}\right| \rightarrow \infty$, respectively.

\section{B. Two-mode squeezed vacuum state}

Two-mode squeezed states are the prototype of nonclassical states of light, having strong quantum correlations between the modes that are responsible for intensity correlations and for the squeezing property itself. They have an impressive list of fundamental properties [32], to which it can be added their beautiful phase properties.

They are defined by applying the two-mode squeeze operator on the vacuum state

$$
|\zeta\rangle=\exp \left(\zeta^{*} a_{1}^{\dagger} a_{2}^{\dagger}-\zeta a_{1} a_{2}\right)|0,0\rangle
$$

and their expression in the number basis is

$$
|\zeta\rangle=\sum_{n=0}^{\infty} \frac{(\tanh s)^{n}}{\cosh s} \exp (i n \xi)|n, n\rangle,
$$

where the squeezing parameter is $\zeta=s \exp (i \xi)$, and they contain only even total photon number states. We can see that their projection on each subspace $\mathcal{H}_{2 n}$ is precisely a number difference eigenstate, so we can expect some uniform distribution in phase difference. The mean value of any $f\left(\Phi_{12}\right)$ is

$$
\left\langle\zeta\left|f\left(\Phi_{12}\right)\right| \zeta\right\rangle=\sum_{n=0}^{\infty} \sum_{r=0}^{2 n} f\left(\phi_{r}^{(2 n)}\right)\left|\left\langle\phi_{r}^{(2 n)} \mid \zeta\right\rangle\right|^{2},
$$

where

$$
\left|\left\langle\phi_{r}^{(2 n)} \mid \zeta\right\rangle\right|^{2}=\frac{(\tanh s)^{2 n}}{(2 n+1) \cosh ^{2} s} .
$$

This last expression, being independent of $r$, shows that on every fixed $\mathcal{H}_{2 n}$ subspace the two-mode squeezed vacuum state can be found with every allowed phase value with the same probability. However, the discrete character, especially relevant when $n=0$, makes the main difference here with other continuous approaches like the one based on the Pegg-Barnett formalism, which gives a continuous uniform phase distribution $[21,33]$. Then, we can expect coincident results for high values of the mean photon number, but differences in the case of small photon numbers.

The expressions for the phase difference and its uncertainty are

$$
\begin{gathered}
\left\langle\Phi_{12}\right\rangle=\phi_{0}+\pi\left\langle\frac{N}{N+1}\right\rangle, \\
\left\langle\left(\Delta \Phi_{12}\right)^{2}\right\rangle=\frac{4 \pi^{2}}{3}\left\langle\frac{N(N+1 / 2)}{(N+1)^{2}}\right\rangle-\pi^{2}\left\langle\frac{N}{N+1}\right\rangle^{2} .
\end{gathered}
$$

The form of the mean values in this equation shows that in the limit of high values of $s$ they tend to 1 , so $\left\langle\left(\Delta \Phi_{12}\right)^{2}\right\rangle \rightarrow \pi^{2} / 3$, as expected for a continuous random variable. When $s$ tends to zero, $\left\langle\left(\Delta \Phi_{12}\right)^{2}\right\rangle \rightarrow 0$. This happens because $|\zeta\rangle$ is then closer to the $|0,0\rangle$ state. In Fig. 2 we have plotted the phase-difference uncertainty as a function of the mean total photon number, showing these two behaviors.

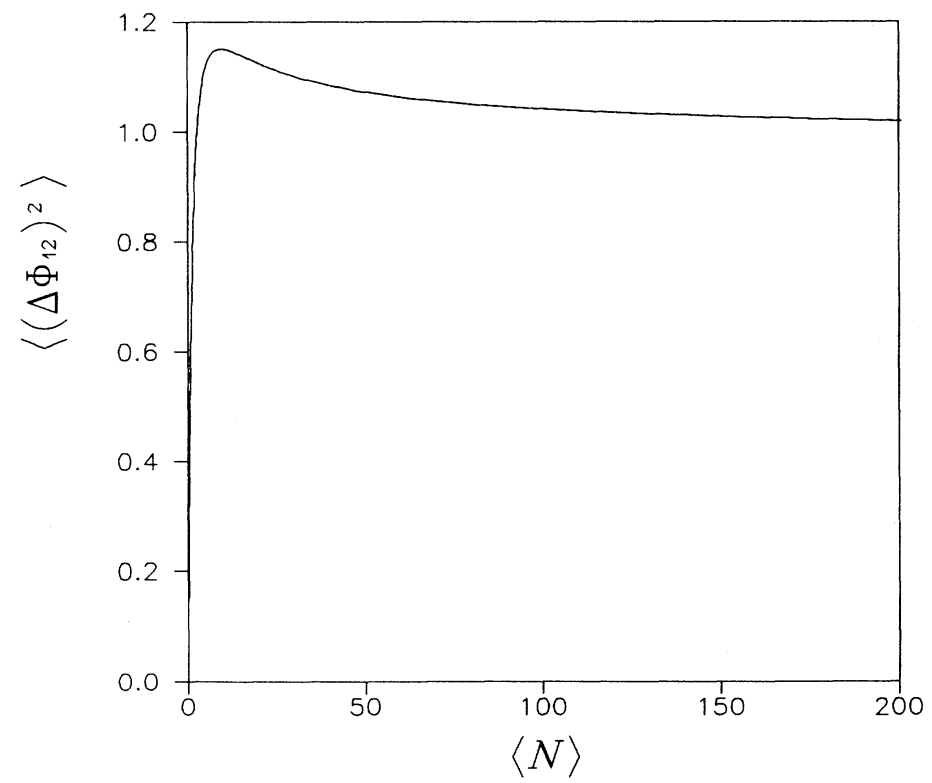

FIG. 2. Variance of the phase difference as a function of the mean total photon number for a two-mode squeezed state. The values of $\left\langle\left(\Delta \Phi_{12}\right)^{2}\right\rangle$ have been normalized to $\pi^{2} / 3$, which is the limit expected for a continuous random variable. 


\section{CONCLUSIONS}

In this paper we have investigated an appropriate operator for the quantum description of the relative phase difference of two modes of the electromagnetic field. We have identified this operator with a proper polar decomposition of the Stokes operators for the system, showing a clear counterpart in the classical limit.

The eigenvalue spectrum of this operator is discrete, having $n+1$ possible values, $n$ being the total number of quanta in the system. We have justified this behavior on the grounds of a phase-sensitive measurement. As expected, in the limit of high $n$ we recover a dense spectrum, and the operator gives the same predictions of other approaches.
The behavior of this operator for some interesting twomode real states has been compared with other operators, finding a good agreement for high number of photons.

\section{ACKNOWLEDGMENTS}

The authors would like to thank Professor A. Galindo and Professor R. Tanaś for a critical reading of the manuscript and useful comments. They are grateful as well to Professor J. F. Cariñena for helpful and enlightening discussions of some rather technical points. Finally, they benefited from the continuous interest and advice of Professor E. Bernabeu.
[1] P. Carruthers and M. M. Nieto, Rev. Mod. Phys. 40, 441 (1968).

[2] S. M. Barnett and D. T. Pegg, J. Phys. A 19, 3849 (1986).

[3] D. T. Pegg and S. M. Barnett, Europhys. Lett. 6, 483 (1988); J. Mod. Opt. 36, 7 (1989).

[4] J. Bergou and B. G. Englert, Ann. Phys. (N.Y.) 209, 479 (1991).

[5] H. Gerhardt, U. Buchler, and G. Litfin, Phys. Lett. 49A, 119 (1974).

[6] D. R. Matthys and E. T. Jaynes, J. Opt. Soc. Am. 70, 263 (1980).

[7] N. G. Walker and J. E. Carrol, Opt. Quantum Electron. 18, 355 (1986).

[8] R. Lynch, Phys. Rev. A 41, 2841 (1990).

[9] C. C. Gerry and K. E. Urbańsky, Phys. Rev. A 42, 662 (1990).

[10] A. Bandilla, Opt. Commun. 80, 267 (1991).

[11] J. W. Noh, A. Fougères, and L. Mandel, Phys. Rev. Lett. 67, 1426 (1991).

[12] L. Susskind and J. Glogower, Physics 1, 49 (1964).

[13] E. C. Lerner, Nuovo Cimento B 56, 183 (1968).

[14] M. Ban, J. Math. Phys. 32, 3077 (1991); Opt. Commun. 94, $231(1992)$ and references therein.

[15] Z. Hradil, Quantum Opt. 4, 93 (1992); Phys. Rev. A 47, 2376 (1993).

[16] Quantum Theory of Angular Momentum, edited by L. C. Biedenharn and H. van Dan (Academic, New York, 1965).

[17] A. Vourdas, Phys. Rev. A 41, 1653 (1990).

[18] D. Ellinas, J. Math. Phys. 32, 135 (1991).
[19] A. Luis and L. L. Sánchez-Soto, Phys. Rev. A 47, 1492 (1993).

[20] J. M. Lévy-Leblond, Rev. Mex. Fis. 22, 15 (1973); Ann. Phys. (N.Y.) 101, 319 (1976).

[21] S. M. Barnett and D. T. Pegg, Phys. Rev. A 42, 6713 (1990).

[22] J. C. Garrison and J. Wong, J. Math. Phys. 11, 2242 (1970); A. Galindo, Lett. Math. Phys. 8, 495 (1984); 9, 263 (1985).

[23] M. Born and E. Wolf, Principles of Optics (Pergamon, Oxford, 1980)

[24] J. M. Jauch and F. Rohrlich, The Theory of Photons and Electrons (Addison-Wesley, Reading, MA, 1959).

[25] R. Tanaś and Ts. Gantsog, Opt. Commun. 87, 369 (1992).

[26] A. I. Akhiezer and V. B. Berestetskii, Quantum Electrodynamics (Interscience, New York, 1965).

[27] H. P. Yuen and J. H. Shapiro, IEEE Trans. Inf. Theory IT-26, 78 (1980).

[28] B. L. Schumaker, Opt. Lett. 9, 189 (1984).

[29] B. Yurke, S. L. McCall, and J. R. Klauder, Phys. Rev. A 33, 4033 (1986).

[30] S. Prasad, M. O. Scully, and W. Martienssen, Opt. Commun. 62, 139 (1987).

[31] R. J. Glauber, Phys. Rev. 131, 2766 (1963).

[32] C. M. Caves and B. L. Schumaker, Phys. Rev. A 31, 3068 (1985); B. L. Schumaker and C. M. Caves, ibid. 31, 3093 (1985).

[33] Ts. Gantsog and R. Tanaś, Phys. Lett. A 152, 251 (1991). 